\title{
EPOC en el adulto mayor: control de síntomas en consulta del Médico de Familia
}

\author{
Ramiro Eduardo Guzmán Guzmánª
}

\begin{abstract}
a Médico Especialista en Medicina Familiar,

Diplomado en Educación Superior y Diplomado en Gestores de Gerontología y Atención Geriátrica en Salud para el Adulto Mayor.

La Paz (Bolivia).
\end{abstract}

\section{Correspondencia:}

Ramiro Eduardo Guzmán

Guzmán. E-mail:

guzman_ramiro@hotmail.com

Recibido el 16 de agosto de 2008.

Aceptado para su publicación el 6 de septiembre de 2008.

\begin{abstract}
RESUMEN
El presente trabajo de revisión se realizó con el propósito de poner a disposición aspectos básicos y elementales de la enfermedad pulmonar obstructiva crónica (EPOC) en el paciente geriátrico. Siendo uno de los procesos patológicos pulmonares por limitación de flujo aéreo asociado a una respuesta inflamatoria anormal a partículas o gases nocivos, no difiere mucho de otros grupos etáreos. La importancia para el medico de familia será mantener la enfermedad estable y proporcionar mejor calidad de vida, tomando en cuenta durante el seguimiento en estos pacientes la aparición de síntomas que muestren estadios avanzados de la enfermedad y el impacto de la enfermedad en la vida cotidiana. Sobre todo se debe indagar el grado de soporte familiar y social de que dispone el paciente. Por lo tanto, el manejo de la EPOC estable esta destinado a la prevención de la enfermedad, alivio de síntomas, prevención y tratamiento de las complicaciones y reagudizaciones. Se debe recordar siempre que la edad avanzada es un factor asociado a mortalidad y aparición de complicaciones, lo cual nos obliga a estar pendientes a una posible hospitalización del paciente en control.
\end{abstract}

Palabras clave. Enfermedad Pulmonar Obstructiva Crónica.

\section{ABSTRACT}

Chronic Obstructive Pulmonary Disease in the elderly: control of symptoms in the GP's clinic The present review was carried out in order to make available basic and elementary aspects of chronic obstructive pulmonary disease (COPD) in the geriatric patient. Being one of the pulmonary pathological processes caused by limitation of air flow associated with an abnormal inflammatory response to harmful particles or gases, it is similar to other age-related clinical conditions. The importance for the family doctor is to maintain the disease stable and to provide a better quality of life, taking into account during this process the appearance of symptoms that show advanced stages of the disease, the impact of the disease on the patient's daily life and the degree of family and social support the patient receives. Therefore, the management of stable COPD is aimed at preventing the disease, relieving symptoms and preventing and treating complications and relapses. It must always be born in mind that advanced age is associated with complications and mortality, which means that we should always be aware of the possible need to hospitalize the patient under control.

Key words. Pulmonary Disease, Chronic Obstructive.

\section{INTRODUCCION}

La enfermedad pulmonar obstructiva crónica es una patología de alta prevalencia a nivel mundial (15\% de la población mundial), de condición progresiva y deterioro significativo en la calidad de vida, que tiene gran impacto económico en las personas y los sistemas de salud ${ }^{1}$. En la población geriátrica existe cifras elevadas de esta patología, debido a que su diagnóstico en consulta de atención primaria de salud no es necesariamente inicial sino que en su mayor porcentaje es de control y seguimiento, es decir de tratamiento crónico. Por lo tanto, la terapia del paciente con EPOC estable debe ser de control directo y exhaustivo por el médico de familia, para llegar no a la cura sino a mejorar la calidad de vida de este paciente con patología pulmonar crónica.

\section{DEFINICIÓN}

Enfermedad prevenible y tratable caracterizada por limitación al flujo aéreo que no es totalmente reversible, de curso progresivo y que se asocia con una respuesta inflamatoria anormal de los pulmones a partículas o gases nocivos, fundamentalmente el tabaco ${ }^{1,2,14,19}$.

Según la SEPAR, la EPOC consiste en una "obstrucción crónica y poco reversible al flujo aéreo (disminución del volumen espiratorio forzado en el primer segundo -FEV1-y de la relación FEV1/capacidad vital forzada -FVC-) causada principalmente por una reacción inflamatoria frente al humo del tabaco"14,19. 


\section{EPIDEMIOLOGÍA.}

Afecta al 15\% de la población mundial. Su incidencia continúa aumentado a pesar de ser una enfermedad que se puede prevenir mediante el abandono del tabaco $^{14,19}$. Según datos de la OMS, se ha calculado que la EPOC será la $3^{a}$ causa de mortalidad mundial en 2020 y ocupara el $2^{\circ}$ lugar entre las causas de morbilidad $^{23}$. En Chile la EPOC representa el $22 \%$ del total de enfermedades respiratorias, siendo la segunda causa de muerte. Anualmente, fallecen entre 1.500 y 1.700 personas por EPOC y en la mayoría de ellas la muerte ocurre sobre los 65 años de edad ${ }^{16}$.

La EPOC en pacientes de altura del Cuzco (Perú) es de presentación temprana, con predominio en la quinta década y en el sexo masculino, con tendencia al sobrepeso y concentración en grupos familiares. Presenta mayor incidencia en grupos laborales y nivel socioeconómico preponderante de tipo medio ${ }^{17,18}$.

\section{FACTORES DE RIESGO14,19}

- Ambientales: tabaquismo (activo y pasivo), infecciones respiratorias durante la infancia, polución atmosférica y exposición laboral a polvos o sustancias químicas.

- Genéticos: genes específicos, déficit de alfa-1antitripsina, hiperreactividad bronquial/atopía y antecedente de bajo peso al nacer.

\section{CLASIFICACIÓN ${ }^{2,19}$}

La Clasificación de la EPOC (GOLD/ATS-ERS), basada en criterios espirométricos, se muestra en la tabla 1.

FACTORES DESENCADENANTES DE LOS EPISODIOS DE EXACERBACIÓN DE LA EPOC ${ }^{21}$

- Causas de exacerbación: infección del árbol traqueobronquial (bacteriana, vírica u otros organismos), contaminación atmosférica o desconocida.

- Enfermedades concomitantes que agravan el estado clínico: enfermedades respiratorias (neumonía, tromboembolismo pulmonar, neumotórax, derrame pleural y traumatismo torácico), alteraciones cardiovasculares (insuficiencia cardiaca congestiva, trastorno del ritmo cardiaco y shock), depresión del centro respiratorio (fármacos antipsicóticos y ansiolíticos y administración de oxigeno en concentraciones elevadas), enfermedades abdominales (cirugía, enfermedades inflamatorias y ascitis) y alteraciones sistémicas (alcalosis metabólica, sepsis y desnutrición).

\section{DIAGNÓSTICO}

- Anamnesis: antecedente de factores de riesgo (tabaquismo, exposición crónica a humo o riesgos ocupacionales $\left.{ }^{1,15}\right)$, antecedente de enfermedad respiratoria previa, enfermedad actual (síntomas habituales agudizados o factores desencadenantes), presencia de comorbilidades asociadas como enfermedades cardiacas que contribuyen a la limitación de la actividad, impacto de la enfermedad en la vida diaria (limitación de la actividad, impacto económico y trastornos afectivos) y grado de soporte familiar y social del paciente.
- Síntomas: tos crónica (puede ser intermitente o diaria, a lo largo del día, pero más intensa por la mañana, y rara vez sólo nocturna), expectoración crónica (puede seguir cualquier patrón), disnea (progresiva, persistente y empeora con el esfuerzo y con infecciones intercurrentes, síntoma tardío que conlleva una progresiva incapacidad funcional que repercute negativamente en la calidad de vida $^{4}$ ), intolerancia al ejercicio (proporcional a las alteraciones de la función pulmonar y al compromiso ventilatorio) y anorexia y pérdida de peso (se presentan en estadios avanzados de la enfermedad y son síntomas indicativos de mal pronóstico).

- Sígnos: cianosis central y periférica, deformidad torácica por hiperinflación pulmonar, sibilancias aisladas en la espiración forzada, roncus inspiratorios, espiración forzada mayor de 6 segundos, disminución de la matidez cardíaca y ruidos cardiorrespiratorios alejados. Con frecuencia presencia de hernias inguinales por aumento de la presión abdominal producida por la espiración forzada y la tos. Si se presenta hemoptisis, es de escasa cantidad; mezclada con el esputo puede presentarse en los episodios de infección bronquial (de todos modos, si es intensa o no se acompaña de mayor producción de esputo, debe considerarse la posibilidad de un tumor broncopulmonar). En fases avanzadas datos clínicos de insuficiencia ventricular derecha (Cor Pulmonale Crónico).

\section{EXÁMENES COMPLEMENTARIOS 5,7,12,15}

- Pruebas de función pulmonar: espirometría (de confirmación diagnostica), volúmenes pulmonares, gasometría arterial basal, oximetría nocturna, capacidad de difusión de monóxido de carbono e intercambio pulmonar de gases.

- Pruebas de esfuerzo: marcha de los 6 minutos.

- Pruebas de imagen: radiografía de tórax (PA y lateral) y TAC de tórax.

- Otras pruebas complementarias: detección de alfa1-antitripsina, hemograma completo (valoración de eritrocitosis, signos de desviación a la izquierda, trombocitosis o trombopenia; valoración de flebotomias), química sanguínea (función renal, equilibrio hidroelectrolítico), niveles plasmático de teofilina en caso de recibir tratamiento con teofilina, electrocardiograma (taquicardia, arritmias, sobrecarga del VD, IAM, TEP), ecocardiograma, espirometría anual en pacientes estables, valoración de la calidad de la vida (Cuestionario de Respiración Crónica y Cuestionario de Enfermedad Respiratoria de George-SGRQ-) y valoración funcional (Índice de Katz de las actividades de la vida diaria e Índice de Lawton de las actividades instrumentales de la vida diaria).

\section{DIAGNÓSTICO DIFERENCIAL 5,15}

- Asma bronquial.

- Insuficiencia cardiaca congestiva.

- Bronquiectasias.

- Síndrome de apnea del sueño.

- Bronquiolitis obliterante. 


\begin{tabular}{l|cc}
\hline \multirow{2}{*}{ Estadio } & \multicolumn{2}{|c}{ Características } \\
\cline { 2 - 3 } & FEV1 (\% del valor teórico) & FEV1/FVC \\
\hline 0: En riesgo* & Espirometria normal & $\geq \ldots 0,7$ \\
I: EPOC leve & $>80$ & $<0,7$ \\
II. EPOC moderada & $50-80$ & $<0,7$ \\
III. EPOC grave & $30-50$ & $<0,7$ \\
IV. EPOC muy grave & $<30$ & $<0,7$ \\
\hline
\end{tabular}

Tabla 1. Clasificación de la EPOC (GOLD/ATS-ERS) basada en criterios espirométricos. *Incluir a pacientes fumadores o que presentan exposición ambiental clara y síntomas como tos, expectoración o disnea. FEV1 Volumen espiratorio forzado en 1 minuto. Fuente:referencia 19.

- Panbronquiolitis difusa.

- Cáncer de pulmón.

- Fibrosis quística.

\section{TRATAMIENTO}

\section{Tratamiento no farmacológico. EPOC leve - mod- erado}

- Oxigenoterapia crónica domiciliaria (>15 horas diarias) $)^{11,19}$. Indicaciones absolutas: $\mathrm{PaO} 2<55$ $\mathrm{mmHg}$ a nivel del mar, $\mathrm{Pa0} 2$ entre 55 y $60 \mathrm{mmHg}$ con evidencia de repercusión orgánica, hipertensión pulmonar, cor pulmonale crónico, insuficiencia cardíaca congestiva, arritmias, hematócrito >55\% y repercusión del intelecto.

- Rehabilitación respiratoria19: educación del paciente, fisioterapia respiratoria, soporte psicosocial y entrenamiento muscular.

- Tratamiento de las alteraciones nutricionales ${ }^{1,19}$ : requerimientos proteicos similares a los de la población general (1,3 y $1,5 \mathrm{~g}$ de proteínas $/ \mathrm{kg} /$ día), evitar el exceso de calorías no proteicas (las dietas pobres en grasas y ricas en hidratos de carbono mejoran la función pulmonar posprandial y la sensación de disnea), recomendar ingesta de ácidos grasos omega 3, que modulan la producción de mediadores inflamatorios y el mecanismo de broncoconstricción y conseguir un adecuado aporte hídrico para favorecer la eliminación de secreciones.

- Recomendaciones ${ }^{1,2,16}$ :

- Abandono del habito tabáquico: de gran importancia en cualquier fase de la enfermedad, demostrándose un menor descenso en el FEV1 y menos síntomas respiratorios al cabo de 5 años del cese del hábito tabaquico.

- Vacunación antigripal anual: la vacunación antigripal anual ha demostrado reducir la gravedad de las exacerbaciones y hospitalizaciones por neumonía y gripe en la población anciana con EPOC, recomendándose el uso de vacunas con virus muertos o inactivados y la revacunación sólo en personas mayores de 65 años que recibieron por alguna indicación especial la primera dosis antes de los 65 años o las personas con alto riesgo de infección neumocócica grave (asplenia, inmunosupresión). Sin embargo, se puede considerar una medida controversial debido a que los adultos mayores no desarrollan suficientes anticuerpos (Ac) ante la vacuna y a partir de los $70,80,90$ años son más propensas a la neumonía con o sin la influenza. Por lo tanto, existe la incógnita de tomar esta recomendación profiláctica en forma habitual, pero es de considerar ante varios estudios como una medida que vale la pena indicar con el fin de conformar una inmunidad colectiva a la enfermedad, evitar la neumonía como complicación, evitar otras enfermedades respiratorias que también pueden ser mortales y prevenir el número de muertes entre la población geriátrica.

- Vacunación anti-neumocócica: medida indicada para la prevención de enfermedades neumocócicas producidas por diferentes serotipos del streptococo neumoniae, que confiere protección durante al menos 9 años, por lo que no se recomienda la revacunación rutinaria en adultos mayores o pacientes inmunodeprimidos. La administración de la vacuna induce una respuesta tipo específica, con aumento del título de anticuerpos dentro de las 2 - 3 semanas siguientes. Existe una respuesta inmunógena suficiente en grupos de riesgo, como son las personas de 65 o más años o con enfermedades crónicas (EPOC, DM2, enfermedades cardiopulmonares). La indicación de una segunda dosis en personas mayores de 65 años será si pasaron al menos 5 años de la primera dosis o la primera dosis se administró antes de los 65 años. Considerar siempre una posible reacción alérgica tras su administración. Cuando la vacuna anti-neumocócica se administra simultáneamente con la vacuna antigripal, no aumentan las reacciones adversas a ninguna de las dos vacunas.

2. Tratamiento farmacológico: EPOC leve moderado $^{3,12,13,19,23}$

- Broncodilatadores: son fármacos capaces de relajar el músculo liso de las vías respiratorias, proporcionando una mejoría a largo plazo en los índices del flujo espiratorio, disminución de la disnea y aumento de la tolerancia al esfuerzo, aun cuando no se produzca un cambio significativo en el FEV1. Algunos broncodilatadores poseen efectos adicionales como la acción estimulante sobre los cilios del epitelio bronquial (agonistas betaadrenérgicos) 
o la mayor resistencia a la fatiga de los músculos respiratorios (teofilina). Se prefiere utilizarlos por vía inhalatoria ya que por vía sistémica provocan mayor efectos secundarios. Por todo esto constituyen el primer paso en el tratamiento farmacológico de la EPOC. En el paciente con EPOC leve se utiliza broncodilatadores de acción corta a demanda para mejorar la disnea y la limitación al ejercicio; si los síntomas no son controlados o el paciente evoluciona a un estadio moderado, se cambia a fármacos de acción prolongada o combinaciones de beta 2 agonistas de acción corta y anticolinérgicos de acción corta como primera medida, y si no se controlan los síntomas o el paciente presenta dos o mas exacerbaciones al año, está indicado el uso de broncodilatadores de acción prolongada. Son 3 categorías farmacológicas las que han demostrado eficacia clínica en la EPOC: beta 2 agonistas, anticolinérigicos y metilxantinas. La combinación de un simpaticomimético con un anticolinérgico es una buena pauta de mantenimiento por suma de efecto broncodilatador.

- Beta 2 agonistas de acción corta. Son fármacos que han demostrado disminuir la disnea y mejorar la FEV1 en pacientes con EPOC. Son los de mayor utilidad debido a su rapidez de acción (15-30 minutos) y vida media (3-6 horas), por lo que pueden ser utilizados tanto a demanda como de forma regular asociados a otros broncodilatadores. Su empleo a demanda está indicado en pacientes poco sintomáticos o con enfermedad leve, siendo útiles en los episodios de exacerbación y como medida adicional ante síntomas ocasionales. En el paciente geriátrico comenzar con dosis mínimas y modificarlas de acuerdo a respuesta clínica, y siempre considerar el empleo de cámaras espaciadoras si existe dificultad para el manejo de aerosoles. Dosis recomendadas: Salbutamol 100 - 200 ug (microgramos) cada $4-6$ horas (inhalación), Terbutalina $250-500$ ug cada 6 horas (inhalación), Fenoterol 100 - 200 ug cada $4-6$ horas (inhalación).

- Beta 2 agonistas de larga duración. Se les ha considerado en varios estudios como fármacos que mejoran la disnea, la función pulmonar y la calidad de vida de estos pacientes, siendo capaces de reducir el número de exacerbaciones que precisan tratamiento con corticoides orales. Por su tiempo de vida media se puede establecer su tratamiento cada 12 horas. Dosis recomendadas: Formoterol 12 - 24 ug cada 12 horas (inhalación), Salmeterol 50 - 100ug cada 12 horas (inhalación).

- Anticolinérgicos de acción corta. El uso de bromuro de ipratropio ha demostrado incremento en el FEV1 y necesidad de menor medicación de rescate; su inicio de acción brondilatadora es más lenta que los beta 2 agonistas de acción rápida, aunque su duración es más prolongada (6-8horas). Dosis recomendada: Bromuro de Ipratropio 40 - 80 ug cada 6 - 8 horas (inhalación).
- Anticolinérgicos de larga duración. Estos fármacos comparados con los anticolinérgicos de acción corta han demostrado incremento del FEV1, la FVC, mejoría de la disnea, mejora en la calidad de vida, necesidad de menor medicación de rescate y menor número de exacerbaciones. Dosis recomendada: Bromuro de Tiotropio 18 ug cada 24 horas (inhalación).

- Metilxantinas. Son fármacos que además de producir broncodilatación poseen cierta actividad antiinflamatoria. Por la estrecha ventana terapéutica que tienen exigen la monitorización de sus niveles plasmáticos cada 6 o 12 meses, tratando de mantener los niveles entre $5-15$ microgramos $/ \mathrm{ml}$. cuando se utiliza teofilina y ajustando la dosis si se utiliza en forma concomitante con macrólidos o quinolonas. De elección en pacientes con CPC descompensado. Para iniciar tratamiento se debe comenzar con una dosis inicial mínima y aumentarla en función de datos clínicos y cinéticos de modo gradual (incremento del 25\%) en intervalos de 2 ó 3 días, hasta alcanzar el efecto terapéutico deseado con la dosis de mantenimiento. En ancianos presenta una eliminación prolongada y se debe tener precaución durante su uso tanto por sus efectos secundarios como por sus interacciones medicamentosas. Dosis recomendadas: Teofilina 200mg. por vía oral cada 12 horas (se puede llegar hasta 400mg. cada 12 horas), Aminofilina (liberación lenta) 225 - 450mg. por vía oral cada 24 horas.

- Glucocorticoides:

- Glucocorticoides orales. Tratamiento a corto plazo. Indicado en las agudizaciones de la EPOC: prednisona $0.5 \mathrm{mg} / \mathrm{kg}$ peso cada 6-8 horas (al tercer día se realiza pauta descendente hasta retirar el corticoide).

- Glucocorticoides inhalados. Se recomiendan en pacientes que hayan respondido positivamente a su administración, ya sea inhalados u orales, en pacientes con prueba broncodilatadora positiva y en pacientes con enfermedad mal controlada y exacerbaciones frecuentes. Una recomendación es utilizar prednisona a dosis de $30 \mathrm{mg}$ cada día durante dos semanas y según respuesta espirométrica obtenida se puede evaluar el uso continuo por vía inhalatoria. Budesonida $400-800$ ug cada 12 horas (inhalación), Propionato de Fluticasona 500 ug cada 12 horas (inhalación).

- Tratamiento combinado. Cuando los síntomas no se controlan con monoterapia y se considera aumentar la dosis del fármaco utilizado, es preferible añadir otro fármaco al tratamiento, ya que la combinación de dos fármacos ha demostrado mejorar los síntomas y la función pulmonar, además de reducir el riesgo de efectos adversos, sobre todo en este grupo etáreo.

- Corticoides inhalados/B2 agonistas de larga duración: Salmeterol/Propionato de Flutica- 
sona 50/500ug cada 12 horas y Formoterol/ Budesonida 9/320ug cada 12 horas.

- Otras combinaciones de gran utilidad son: beta 2 agonistas + anticolinérgicos, beta 2 agonistas + teofilina o anticolinérgicos + teofilina.

- Tratamiento antibiótico. Se iniciará tratamiento empírico cuando se presenten al menos dos de los siguientes datos clínicos: aumento de la disnea, incremento del volumen de la expectoración y/o aumento de la purulencia del esputo. Para la elección del antibiótico de inicio se puede clasificar al paciente en uno de los siguientes grupos:

- Grupo l: EPOC leve, menor de 65 años y sin comorbilidad. El tratamiento debe cubrir $\mathrm{H}$. Influenzae, S. Pneumoniae y M. Catarrhalis. Tratamiento: Amoxicilina/Ac. Clavulanico $875 / 125 \mathrm{mg}$ por vía oral cada 8 horas por $7-10$ días ó Levofloxacino 500 mg. por vía oral cada 24 horas por 5 días ó Moxifloxacino 400 mg. por vía oral cada 24 horas por 5 días ó Telitromicina 800 mg. por vía oral cada 24 horas por 5 días ó Claritromicina 500mg. por vía oral cada 12 horas o en una toma por 7 dias.

- Grupo lla: EPOC moderada o grave, sin factores de riesgo para P. Aeruginosa. Además de cubrir las bacterias del grupo I, hay que añadir enterobacterias (E. Coli, K. Pneumoniae). Se utiliza el mismo esquema de tratamiento que en el grupo I. Amoxicilina/Ácido clavulanico $875 / 125 \mathrm{mg}$. por vía oral cada 8 horas por 10 días asociado a un macrólido (Azitromicina 500 mg. por vía oral cada 24 horas por 5 dias ó Claritromicina 1000 mg. por vía oral cada 24 horas por 10 días). En pacientes hospitalizados cefalosporinas por vía parenteral (Cefotaxima, Ceftriaxona).

- Grupo llb: EPOC moderada o grave, con factores de riesgo para P. Aeruginosa. Ciprofloxacina 750 mg. por vía oral cada 12 horas por $7-10$ días. En pacientes hospitalizados tratamiento por vía parenteral (Cefepima, Imipenem, Meropenem o Piperacilina/Tazobactam) asociado a fluoroquinolona antipseudomónica o a un aminoglucósido.

- Tratamiento antidepresivo. La depresión es una patología de alta prevalencia en pacientes geriátricos con EPOC y que se debe indagar aún más en pacientes con nivel de saturación de $\mathrm{O} 2$ inferior al $92 \%$, disnea severa o que hayan requerido ingreso hospitalario. El tratamiento es el convencional.

- Otros tratamientos farmacológicos: tratamiento con alfa-1-antitripsina, agentes mucolíticos como Ambroxol, Carbocisteína, $\mathrm{N}$ - acetilcisteina o Yodopropilidenglicerol (tratamiento a considerar en pacientes con tos productiva crónica, que se puede mantener de forma prolongada si disminuye la frecuencia de tos y la producción de esputo. Se ha demostrado que la terapia mucolítica reduce el riesgo de exacerbaciones y el riesgo de hospitalización), agentes antioxidantes ( $\mathrm{N}$ - acetilcisteina), antitusígenos y opiáceos (morfina).

\section{Tratamiento ambulatorio de las exacerbaciones 12,19, 22, 23}

Las exacerbaciones constituyen la causa mas frecuente de visitas médicas, hospitalización y muerte en pacientes con EPOC. Es un evento dentro de la evolución natural de la EPOC, el cual se caracteriza por el aumento de la disnea, tos y/o expectoración, mayor que su variabilidad habitual y que son de intensidad tal que motivan cambios en el tratamiento.

El tratamiento ambulatorio se basara en:

- Evaluar al severidad de la crisis (reconocer riesgo vital).

- Identificar comorbilidades descompensadas.

- Administrar oxigeno a flujos bajos.

- Broncodilatadores. Se aumentará la dosis y frecuencia de administración de broncodilatadores de acción corta. Como los anticolinérgicos tienen menos efectos secundarios y son mejor tolerados que los beta 2 agonistas, se recomienda utilizarlos primero y cuando se han alcanzado las dosis máximas del fármaco elegido puede ser útil añadir el segundo fármaco broncodilatador. Si los pacientes estaban en tratamiento con beta 2 adrenérgicos de larga duración (Salmeterol o Formoterol) solos o en combinación con corticosteroides inhalados, se administrará la dosis máxima recomendada: Salmeterol 50ug/12horas o Formoterol 9ug/12 horas. Si el paciente estaba en tratamiento con Tiotropio no se debe modificar su pauta de administración.

- Glucocorticosteriodes. No hay evidencia suficiente acerca de la eficacia de los corticosteroides inhalados en el tratamiento de las agudizaciones. Si el paciente estaba tomando corticoides en monoterapia o como terapia combinada con un beta 2 adrenérgico, se administrará la dosis máxima recomendada: Fluticasona 500 ug cada 12 horas o Budesonida 320 ug cada 12 horas. El tratamiento con glucocorticoides orales se ha mostrado eficaz en reducir la duración de los síntomas de agudización y el número de recaídas. Aumenta la función pulmonar durante las primeras 72 horas de la exacerbación y no hay evidencia que este beneficio se mantega por mas tiempo. Se recomienda el tratamiento con corticoides en pacientes con un FEV $1<50 \%$ del teórico en tandas de un máximo de 10-15 días a una dosis de 0,5 mg/ $\mathrm{kg} /$ día de Metilprednisolona o equivalente en una única toma diaria.

- Antibióticos. Evaluar criterios para administración de antibióticos.

- Luego de haber realizado las medidas necesarias en el tratamiento de la exacerbación, la observación debe mantenerse durante al menos 2 horas, hasta demostrar la respuesta terapéutica o la indicación de hospitalización. Al alta de la crisis se debe: entregar indicaciones terapeuticas por 
escrito, revisar y educar en la técnica inhalatoria, asegurar la disponibilidad de medicamentos y referir a control de seguimiento en consulta externa.

\section{PREVENCION DE LAS AGUDIZACIONES DE LA EPOC $1,5,19$}

- De eficacia demostrada:

- Dejar de fumar.

- Optimizar el tratamiento de la EPOC en fase estable.

- Tratamiento con corticoides inhalados en pacientes con FEV1 <50\%.

- Vacunación antigripal.

- Antibióticos: Como medida profiláctica no hay evidencias suficientes para recomendar su uso en el paciente con EPOC estable o prevenir las exacerbaciones; sin embargo, en pacientes con enfermedades pulmonares crónicas asociadas a EPOC podría considerarse el uso de antibióticos orales por 7 días cada mes con: Amoxicilina $500 \mathrm{mg}$ via oral cada 8 horas ó Sulfametoxazol/Trimetoprim 800/160mg. por vía oral cada 12 horas ó Doxiciclina 100mg por via oral cada 12 horas.

- Tratamiento con corticoides orales en las agudizaciones.

- Rehabilitación respiratoria.

- Educación sanitaria, plan de autocuidados de la enfermedad.

- De eficacia probable:

- Vacunación antineumocócica.

- Inmunomoduladores.

- De eficacia cuestionada

- Antioxidantes.

- Mucolíticos.

\section{CRITERIOS DE DERIVACION ${ }^{15,19,22}$}

- Exacerbación aguda con aumento de la disnea, tos, expectoración purulenta y al menos unos de los siguientes:

- No respuesta a tratamiento ambulatorio bien realizado.

- Incapacidad para realizar tratamiento.

- Dependiente en evaluación de AVD y AIVD.

- Falta de apoyo familiar.

- Comorbilidad de alto riesgo.

- $\quad$ Alteración del nivel de conciencia.

- Aparición o empeoramiento de cor pulmonale.

- Insuficiencia respiratoria crónica.

- Neumotórax.

- Embolismo pulmonar.

- Tratamiento quirúrgico:

- Drenaje intrabulloso.

- Bullectomía mediante toracoscopia o cirugía abierta.

- Cirugía de reducción de volumen pulmonar.

- Trasplante pulmonar.

\section{BIBLIOGRAFÍA}

1. Agustí AG. La EPOC como enfermedad sistémica. En: De Lucas Ramos P, Peces-Barba Romero G, editores. Enfermedad pulmonar obstructiva crónica. Madrid: Editor S.A.; 2001. p. 53-72.

2. Global strategy for the diagnosis, management, and prevention of chronic obstructive pulmonary disease. NHLBI/WHO Global Initiative for Chronic Obstructive Lung Disease (GOLD). Update 2003. Disponible en: http://www.goldcopd.com

3. Standards for the diagnosis and treatment of patients with COPD: a summary of the ATS/ERS position paper. Eur Respir J. 2004; 23:932-6.

4. Hogg JC. Pathophysiology of airflow limitation in chronic obstructive pulmonary disease. Lancet. 2004; 364:709-21.

5. Barberà JA, Peces-Barba G, Agustí AGN, Izquierdo JL, Monsó F, Montemayor T, et al. Guía clínica para el diagnóstico y el tratamiento de la enfermedad pulmonar obstructiva crónica. Arch Bronconeumol. 2001; 37:297-326.

6. Casanova Macario C, García-Talavera Martín I, De Torres Tajes JP. La disnea en la EPOC. Arch Bronconeumol. 2005; 41:2432.

7. Perpiñá Tordera M, Lloris Bayo A. Repercusión de la EPOC sobre el estado de salud. Arch Bronconeumol. 2005; 41:33-8.

8. Marín Trigo JM, Sánchez Barón A. Valoración funcional del paciente con EPOC. Arch Bronconeumol. 2005; 41:18-23.

9. British Thoracic Society Standards of Care Subcommittee on Pulmonary Rehabilitation. Pulmonary rehabilitation. Thorax. 2001; 56:827-34.

10. Hill NS. Noninvasive ventilation has been shown to be ineffective in stable COPD. Am J Respir Crit Care Med. 2000; 161:68990.

11. Wijkstra PJ, Lacasse Y, Guyatt GH, Casanova C, Gay PC, Meecham Jones $\mathrm{J}$, et al. A meta-analysis of nocturnal noninvasive positive pressure ventilation in patients with stable COPD. Chest 2003; 124:337-43.

12. Miravitlles M. Enfermedad pulmonar obstructiva crónica, diagnóstico y tratamiento. Med Clin (Barc). 2005; 125:65-74.

13. Blanquer D, Sala E. Farmacología de la enfermedad pulmonar obstructiva. Medicine 2006; 9(62):4097 - 4104.

14. Balaguer C, Carrera M, Agusti A. Enfermedad pulmonar obstructiva cronica (I). Medicine. 2006; 9 (63):4077 - 4079.

15. Palou A, Sala E. Enfermedad pulmonar obstructiva cronica (II). Medicine. 2006; 9(63):4080 - 4087.

16. Amigo C, Erazo B, Oyarzum M, Manuel et al. Tabaquismo y enfermedad pulmonar obstructiva crónica: determinación de fracciones atribuibles. Rev. Méd. Chile. 2006; 134(10):12751282.

17. Valiente O, Oswaldo V, Uldarico A, Soledad R, Pezo P. EPOC: características clínicas, epidemiológicas y radiológicas en pacientes de altura-hospital es salud de Cuzco (1987 - 1999). Enfermedades del Torax. 2000; 43(1):567-574.

18. Palmero E. Cor pulmonale cronico: causas, enfermedades asociadas y caracteristicas evolutivas en 2172 pacientes internados, Hospital Obrero $n^{\circ}$ 1, La Paz, Bolivia. Latido 2002; 5(6).

19. Blanco AJ, Blázquez JL, Boyano I. EPOC. COR PULMONALE. En: Sociedad Española de geriatría y gerontología. Tratado de geriatria para residentes. Segunda Edición. Barcelona; 2005. p. $371-379$.

20. Gutierrez J. Corticoides inhalados en el paciente EPOC. Revista Electrónica de Medicina Intensiva 2001; 1(10).

21. Barberá JA, Cosio MG. Enfermedad pulmonar obstructiva crónica. En: Aguado JM, Aguilar JL, Aguirre C, Agusti C, Agusti A, De Alarcón A, editores. Farreras Rozman: Medicina interna: 15ed. Madrid - España: Fareso; 2004. p. 740-751.

22. Ministerio de Salud de Chile. Guía clínica de enfermedad pulmonar obstructiva cronica de tratamiento ambulatorio. Santiago: Minsal; 2006.

23. Laredo LM. Farmacos en el tratamietno de la enfermedad obstructiva crónica. En: Lorenzo P, Morena A, Loza JC, Lizardian I, Moro MA. Editores. Farmacología básica y clínica. $17^{a}$ edición. Madrid: Editorial Panamericana; 2005. p.907-918. 